\title{
Numerics of Discrete Element Simulations in Milli-g Environments: Challenges and Solutions
}

\author{
F. Buchele ${ }^{\dagger}$, R. Lichtenheldt ${ }^{\dagger}$ and L. Stubbig ${ }^{\dagger}$ \\ ${ }^{\dagger}$ Institute of System Dynamics and Control, German Aerospace Center (DLR) \\ Münchener Straße 20, 82234 Weßling, Germany \\ buchelefelix@gmail.com, roy.lichtenheldt@dlr.de, leon.stubbig@dlr.de
}

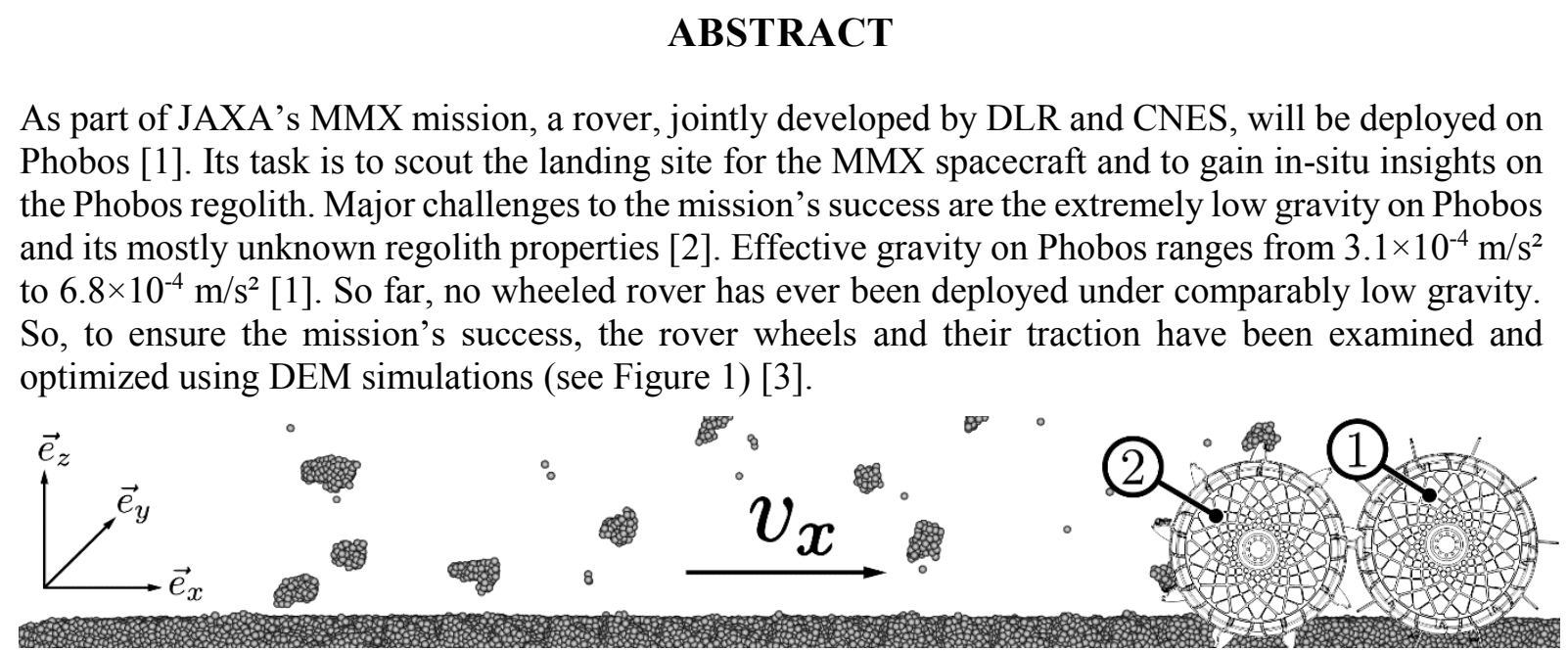

Figure 1: comparison of optimized (1) and non-optimized (2) wheel geometry in partsival

However, the DEM simulation framework, partsival, was developed with the gravitational environments of Earth, Mars, and Moon in mind. Under such conditions, partsival is capable of replicating wheel-soil-interactions. The results of simulations match with experimental findings, so partsival can be considered a well-tested and well-working simulation framework [4]. Minor result scattering is a well-known issue with massively parallelized computations [5]. partsival's simulation results are only non-deterministic on a microscopic scale. Macroscopic determinism is given [4].

However, we observed problems with repeatability when running simulations in Phobos' low gravity environment [3]. When simulating with tried and tested settings, the results show a wide scatter with over $100 \%$ result deviation from lowest to highest slip/sinkage results. Macroscopic determinism is hence clearly not given. Therefore, a method to minimize result scattering with DEM simulations in milli-g environments was developed.

The scattering results emerge from a combination of rounding errors due to parallel computation of inter-particle dynamics [5] and low contact forces (due to Phobos' low gravity) ranging well within the error tolerance of partsival's solver [3]. Several ways to tackle result scattering in milli-g environments are discussed in the paper, including statistical analysis, changes to partsival's numerical precision, and changes to the integration scheme. The numerical stability of the simulation framework is discussed as well and the methods that were found to increase numerical stability in milli-g environments are presented.

\section{REFERENCES}

[1] S. Tardivel, C. Lange, The MMX rover: An innovative Design enabling Phobos in-situ Exploration, LCPM Low-Cost Planetary Missions Conference, IAA, 2019.

[2] S. Ulamec, et al, A rover for the JAXA MMX Mission to Phobos, IAC International Astronautical Congress, IAF, 2019.

[3] F. Buchele, R. Lichtenheldt, Multi-Parameter Rover Wheel and Grouser Optimization for Deployment in Phobos' Milli-g Environment, i-SAIRAS International Symposium on Artificial Intelligence, Robotics and Automation in Space, LPSC, 2020.

[4] R. Lichtenheldt, et al, partsival - Collision-based Particle and many-body Simulations on GPUs for Planetary Exploration Systems, IMSD Joint International Conference on Multibody System Dynamics, IDMEC, 2018.

[5] N. Kapre, A. De Hon, Optimistic Parallelization of Floating-Point Accumulation, ARITH Symposium on Computer Arithmetic, IEEE, 2007. 